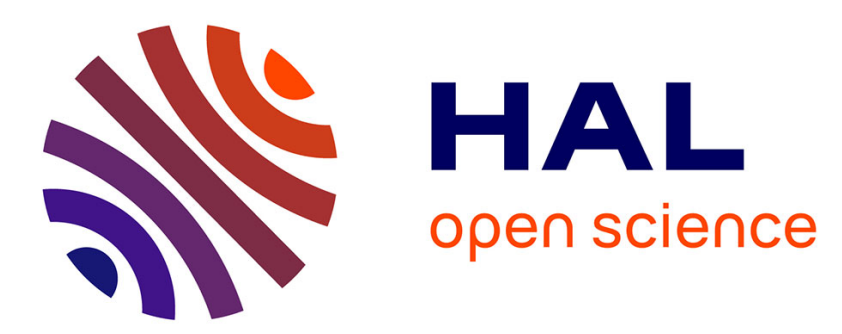

\title{
Sipuleucel-T associated inflammatory cardiomyopathy: a case report and observations from a large pharmacovigilance database
}

Melissa Y.Y. Moey, Rahim A Jiwani, Kotaro Takeda, Karyn Prenshaw, R Wayne Kreeger, John Inzerillo, Darla K Liles, C Bogdan Marcu, Bénédicte Lebrun-Vignes, D Lynn Morris, et al.

\section{To cite this version:}

Melissa Y.Y. Moey, Rahim A Jiwani, Kotaro Takeda, Karyn Prenshaw, R Wayne Kreeger, et al.. Sipuleucel-T associated inflammatory cardiomyopathy: a case report and observations from a large pharmacovigilance database. ESC Heart Failure, 2021, 10.1002/ehf2.13400 hal-03217725

\section{HAL Id: hal-03217725 \\ https://hal.sorbonne-universite.fr/hal-03217725}

Submitted on 5 May 2021

HAL is a multi-disciplinary open access archive for the deposit and dissemination of scientific research documents, whether they are published or not. The documents may come from teaching and research institutions in France or abroad, or from public or private research centers.
L'archive ouverte pluridisciplinaire HAL, est destinée au dépôt et à la diffusion de documents scientifiques de niveau recherche, publiés ou non, émanant des établissements d'enseignement et de recherche français ou étrangers, des laboratoires publics ou privés. 


\title{
Sipuleucel-T associated inflammatory cardiomyopathy: a case report and observations from a large pharmacovigilance database
}

\author{
Melissa Y.Y. Moey ${ }^{1 *}$ (D), Rahim A. Jiwani ${ }^{2}$ (D) Kotaro Takeda ${ }^{3}$, Karyn Prenshaw $^{3}$, R. Wayne Kreeger ${ }^{1}$, \\ John Inzerillo ${ }^{4}$, Darla K. Liles ${ }^{5}$ (D) C. Bogdan Marcu ${ }^{1}$ (D), Bénédicte Lebrun-Vignes ${ }^{6,7}$ (D), D. Lynn Morris ${ }^{1}$, \\ Sivakumar Ardhanari ${ }^{1}$ and Joe-Elie Salem ${ }^{6,8,9}$ \\ ${ }^{1}$ Department of Cardiovascular Sciences, East Carolina Heart Institute, Vidant Medical Center/East Carolina University, 115 Heart Drive, Greenville, NC 27834, USA; \\ ${ }^{2}$ Department of Internal Medicine, Vidant Medical Center/East Carolina University, Greenville, NC, USA; ${ }^{3}$ Department of Pathology and Laboratory Medicine, Vidant Medical \\ Center/East Carolina University, Greenville, NC, USA; ${ }^{4}$ Marion L. Shepard Cancer Center, Washington, NC, USA; ${ }^{5}$ Department of Hematology and Oncology, Vidant Medical \\ Center/East Carolina University, Greenville, NC, USA; ${ }^{6}$ Department of Pharmacology, Regional Pharmacovigilance Centre, Pitié-Salpêtrière Hospital, Sorbonne Université, \\ INSERM CIC-1901, AP-HP, Paris, France; ${ }^{7}$ EA Epiderme-Epidemiology in Dermatology and Evaluation of Therapeutics, Université Paris-Est Créteil, Créteil, France; ${ }^{8}$ UNICO- \\ GRECO APHP.Sorbonne Cardio-Oncology Program, Sorbonne Université, Paris, France; and ${ }^{9}$ Division of Cardiovascular Medicine, Cardio-Oncology Program, Vanderbilt \\ University Medical Center, Nashville, TN, USA
}

\section{Abstract}

Aims The major cardiovascular (CV) adverse effects observed with sipuleucel-T from large multi-institutional clinical trials included thromboembolic events, myocardial infarction, and congestive heart failure in up to $0.3 \%$ of patients with $\mathrm{CV}$ risk factors. The incidence, outcomes, and mechanisms in real-world clinical settings of these CV adverse effects to date have not been fully elucidated. Our study identified a patient with sipuleucel-T-induced inflammatory cardiomyopathy, which led to the identification of CV adverse effects associated with sipuleucel-T from a large pharmacovigilance database and elucidation of its potential mechanisms.

Methods and results Using the MedDRA term 'cardiac disorders' (System Organ Class level), CV adverse events associated with sipuleucel-T versus all other drugs were reviewed from VigiBase, a large pharmacovigilance database. Disproportionality analysis was calculated by the information component (IC), a Bayesian disproportionality indicator. A positive $I_{025}$ (IC 95\% lower end credibility interval) value (>0) is the traditional threshold used in statistical signal detection at the Uppsala Monitoring Centre. From VigiBase, the total number of CV adverse drug reaction reported with sipuleucel-T was 306 out of a total of 22980104 adverse drug reactions in VigiBase on 10/25/2020. MedDRA preferred terms levels were grouped into major CV adverse drug reaction categories where we observed significant reports of myocardial ischaemia, supraventricular tachycardia (particularly atrial fibrillation/atrial flutter), congestive heart failure, and valvular disorders. Myocardial ischemia included acute myocardial infarction $\left(I_{025} 2.3\right)$ with $n=4 / 26(15 \%)$ of these individual case safety reports considered fatal. Among patients with 'cardiac failure congestive' (IC 0251.5$), 11$ of these 43 cases $(26 \%)$ were fatal with $42(98 \%)$ of these cases considered to be solely due to sipuleucel-T.

Conclusions Patients with CV risk factors who are receiving sipuleucel-T may be at higher risk for congestive heart failure, myocardial ischemia, and supraventricular tachycardia. Electrocardiograms during weekly sipuleucel-T infusions and left ventricular function monitoring with echocardiogram should be considered in these patients. Our findings are suggestive of another rare presentation of T-cell-mediated CV toxicity with cancer immunotherapy.

Keywords Prostate cancer; Immunotherapy; Sipuleucel-T; Cardiomyopathy; Cardiotoxicity; Cardio-oncology

Received: 18 August 2020; Revised: 25 February 2021; Accepted: 22 April 2021

*Correspondence to: Melissa Y. Y. Moey, MD MSc, Department of Cardiovascular Sciences, East Carolina Heart Institute, Vidant Medical Center/East Carolina University, 115 Heart Drive, Greenville, NC 27834, USA. Tel: +1 252-744-4400. Email: moeym16@ecu.edu 


\section{Background}

Sipuleucel-T, marketed as Provenge ${ }^{\circledR}$, is an autologous cellular immunological agent ${ }^{1}$ indicated in the treatment of metastatic castration-resistant prostate cancer (mCRPC). ${ }^{2}$ Cardiovascular (CV) toxicities with sipuleucel-T are uncommon $(0.3 \%){ }^{3}$ We report the first case of sipuleucelT-induced inflammatory cardiomyopathy and describe the potential mechanisms. Through review of an international pharmacovigilance database, VigiBase, we also identified increased reporting of sipuleucel-T-associated CV toxicities.

\section{Aims}

In this study, we aimed to elucidate the mechanisms of sipuleucel-T-associated cardiomyopathy and to identify CV adverse effects associated with sipuleucel-T in the real world from VigiBase, the World Health Organization pharmacovigilance database.

\section{Clinical case}

A 64-year-old Caucasian male with well-controlled hypertension managed with lisinopril $10 \mathrm{mg}$ daily was diagnosed with $\mathrm{mCRPC}$ in 2018. He received two doses of subcutaneous degarelix and underwent subsequent transurethral resection of his prostate followed by 6 cycles of docetaxel $75 \mathrm{mg} / \mathrm{m}^{2}$ every 3 weeks along with pegfilgrastim completed 5 months later. He was initiated on enzalutamide $160 \mathrm{mg}$ daily and quarterly intramuscular injections of leuprolide $40 \mathrm{mg}$ shortly after. He had no complications with chemotherapy or hormonal treatment and had significant improvement with prostate-specific antigen (PSA) $<0.1 \mathrm{ng} / \mathrm{mL}$ on subsequent follow-up (Figure 1).

On 6 months of follow-up, he was started on sipuleucel-T every 2 weeks for a total of three infusions. Twenty-four hours after his second infusion of sipuleucel-T, he presented with dyspnea and chest pressure. On physical examination, he was afebrile, blood pressure of $145 / 82 \mathrm{mmHg}$, and heart rate of 74 b.p.m. with an oxygen saturation of $98 \%$ on room air. He had bilateral basilar rales, jugular venous pulse noted to be at least $8 \mathrm{~cm}$ with a positive hepatojugular reflex, and a grade 2/6 pansystolic murmur in the apical region without any radiation. He was warm without any lower extremity edema. Electrocardiogram showed new nonspecific intraventricular conduction delay and non-specific $T$ wave changes in V4 to V6. Troponin I peaked at $0.80 \mathrm{ng} / \mathrm{mL}$ (normal $<0.03 \mathrm{ng} / \mathrm{mL}$ ). Brain natriuretic peptide was $1062 \mathrm{pg} / \mathrm{mL}$ (normal $<200 \mathrm{pg} / \mathrm{mL}$ ).

A transthoracic echocardiogram revealed a left ventricular ejection fraction (LVEF) of 36\%, grade II diastolic dysfunction, grade II mitral regurgitation due to dilated annulus, and mild aortic regurgitation. He received intravenous furosemide $40 \mathrm{mg}$ daily with improvement in symptoms. Left heart catheterization showed $20 \%$ non-obstructive disease in the left anterior descending artery, left circumflex, and right coronary artery. Cardiac magnetic resonance imaging (cMRI) showed biatrial enlargement, moderate mitral regurgitation, and a dilated left ventricular with increased myocardial mass index $\left(116 \mathrm{~g} / \mathrm{m}^{2}\right.$, normal range: $42-85 \mathrm{~g} / \mathrm{m}^{2}$ ) and global hypokinesis with an left ventricular ejection fraction (LVEF) of $32 \%$. There was evidence of late gadolinium enhancement with a mottled mid-wall pattern in the distal septum and the basal inferolateral segment, which was suggestive of a nonischemic cardiomyopathy representing myocardial edema or interstitial fibrosis suspicious for chemotherapy-related cardiomyopathy or infiltrative cardiomyopathy from amyloidosis (Figure 2).

The patient was discharged with a LifeVest and guideline-directed medical therapy for heart failure (HF) with reduced ejection fraction, which included carvedilol $3.125 \mathrm{mg}$ twice a day and lisinopril $20 \mathrm{mg}$ daily with follow-up in the Cardio-Oncology clinic. The patient received his last sipuleucel-T infusion 4 weeks after discharge. During this time, given the concern for cardiac amyloidosis based on cMRI, he was worked up as an outpatient with negative fat pad biopsy, bone marrow biopsy, and urine protein electrophoresis. Serum protein electrophoresis showed elevated serum kappa light chains however with normal kappa to lambda ratio. A nuclear study based on transthyretin protein protocol performed was equivocal for cardiac amyloidosis. He presented 4 months later with a 2 week history of orthopnea found to have new-onset atrial fibrillation and in acute decompensated HF.

An endomyocardial biopsy (EMB) was subsequently performed due to recurrent $\mathrm{HF}$ admission and concern for amyloidosis. The EMB showed several foci of predominant lymphocytic inflammation (Figure 3). Given possible immunotherapy-related myocarditis as observed with immune checkpoint inhibitors $(\mathrm{ICls}),{ }^{4}$ specific staining for T-cell subtypes $\mathrm{CD}^{+}$and $\mathrm{CD}^{+}$was obtained in addition to a non-standard of care stain for programmed cell death ligand-1 (PD-L1), which is overexpressed in $\mathrm{ICl}$-related myocarditis. ${ }^{4}$ Cardiac amyloidosis was also evaluated with Congo red. Haematoxylin and eosin staining showed several foci of mature lymphocytic infiltration (Figure $3 A$ and $3 B$ ) that were positive for both $\mathrm{CD}^{+}$and $\mathrm{CD}^{+}$(Figure 3D-3F). PD-L1 was not detected (Figure 3F). Congo red was negative (Figure 3C). He was discharged on carvedilol $6.125 \mathrm{mg}$ twice a day, lisinopril $20 \mathrm{mg}$ daily, furosemide $20 \mathrm{mg}$ daily, and apixaban $5 \mathrm{mg}$ twice a day. He did not have any recurrent hospitalizations. A repeat echocardiogram 1 month later showed persistent LVEF depression with an improvement to $45-50 \%$ at 6 months of follow-up. 
Figure 1 Timeline of patient diagnosis and treatment of metastatic castration-resistant prostate cancer (mCRPC) and hospitalizations. Our patient was diagnosed with stage IVB prostate cancer in fall of 2018. He was subsequently started on degarelix, which was eventually discontinued due to side effects. He received 6 cycles of docetaxel and started on enzalutamide along with leuprolide. The patient eventually received sipuleucel-T 8 months following his initial diagnosis for mCRPC. Twenty-four hours following his second infusion of sipuleucel-T, he presented to the emergency department with new onset systolic dysfunction (Hospital Admission \#1). He underwent coronary angiogram, which did not show obstructive disease. Cardiac magnetic resonance imaging showed late gadolinium enhancement suggestive of an infiltrative disorder. As an outpatient, he was worked up for amyloidosis, which was negative. He was discharged on lisinopril and carvedilol but presented 3 months later with recurrent symptoms and new onset atrial fibrillation (Hospital Admission \#2). A myocardial biopsy was obtained during his second admission, which showed chronic myocardial inflammation. The patient was treated medically with lisinopril and carvedilol and continues to tolerate enzalutamide, leuprolide, and denosumab without any recurrent hospitalizations. ADHF, acute decompensated heart failure; AF, atrial fibrillation; HFrEF, heart failure with reduced ejection fraction; NSTEMI, non-ST elevation myocardial infarction; Q3w, every 3 weeks; Q6 mo, every 6 months; Qd, every day/daily; SPEP, serum protein electrophoresis; SQ subcutaneous; UPEP, urine protein electrophoresis.

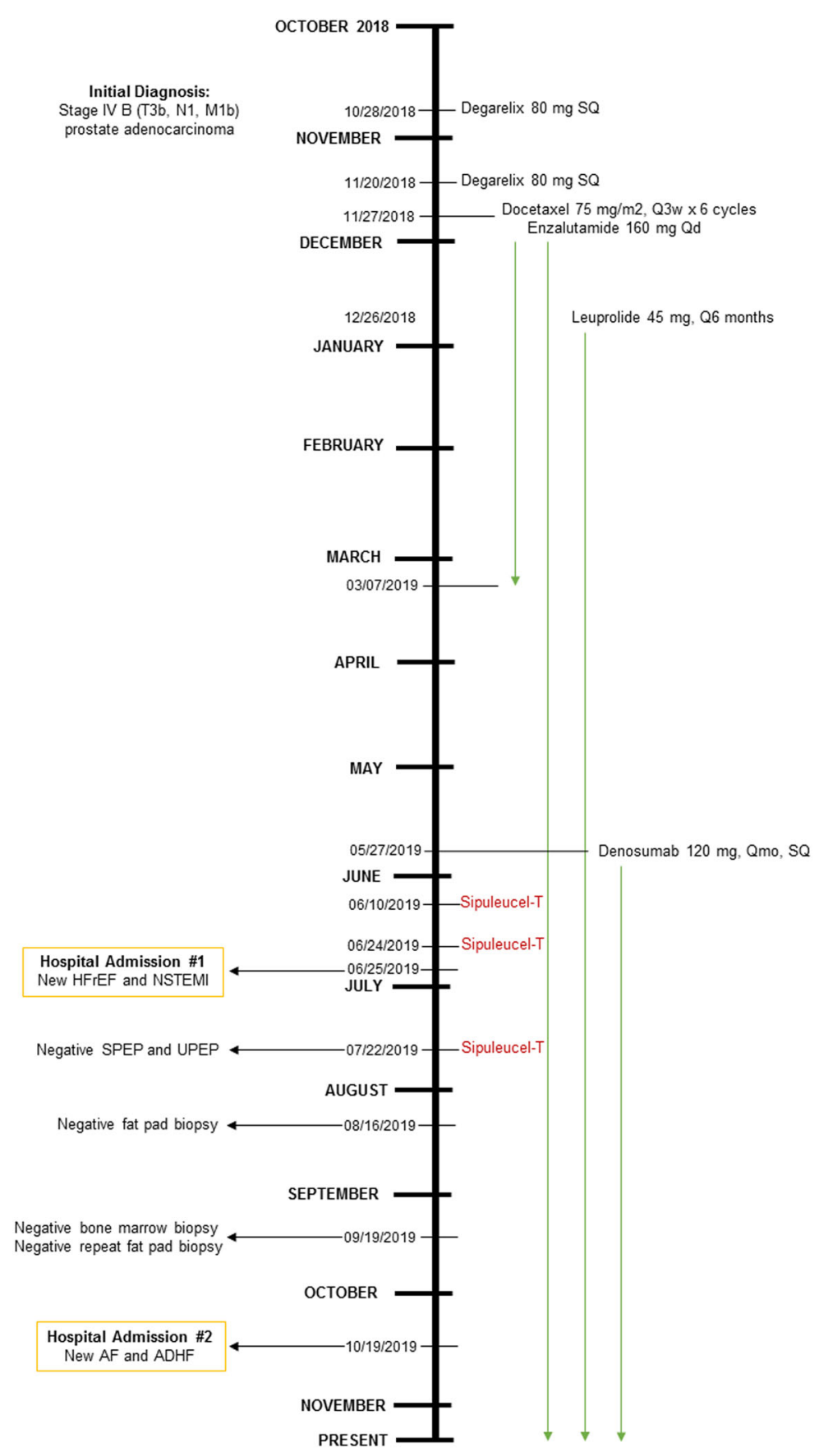


Figure 2 Cardiac magnetic resonance imaging. (A) Three-chamber view. Left panel (magnitude) and right (phase sensitive inversion recovery late gadolinium contrast) study demonstrating increased signal intensity in the inferolateral and distal septal regions. (B) Short-axis view. There is mottled increased signal intensity on phase sensitive inversion recovery sequence in the distal septal area.
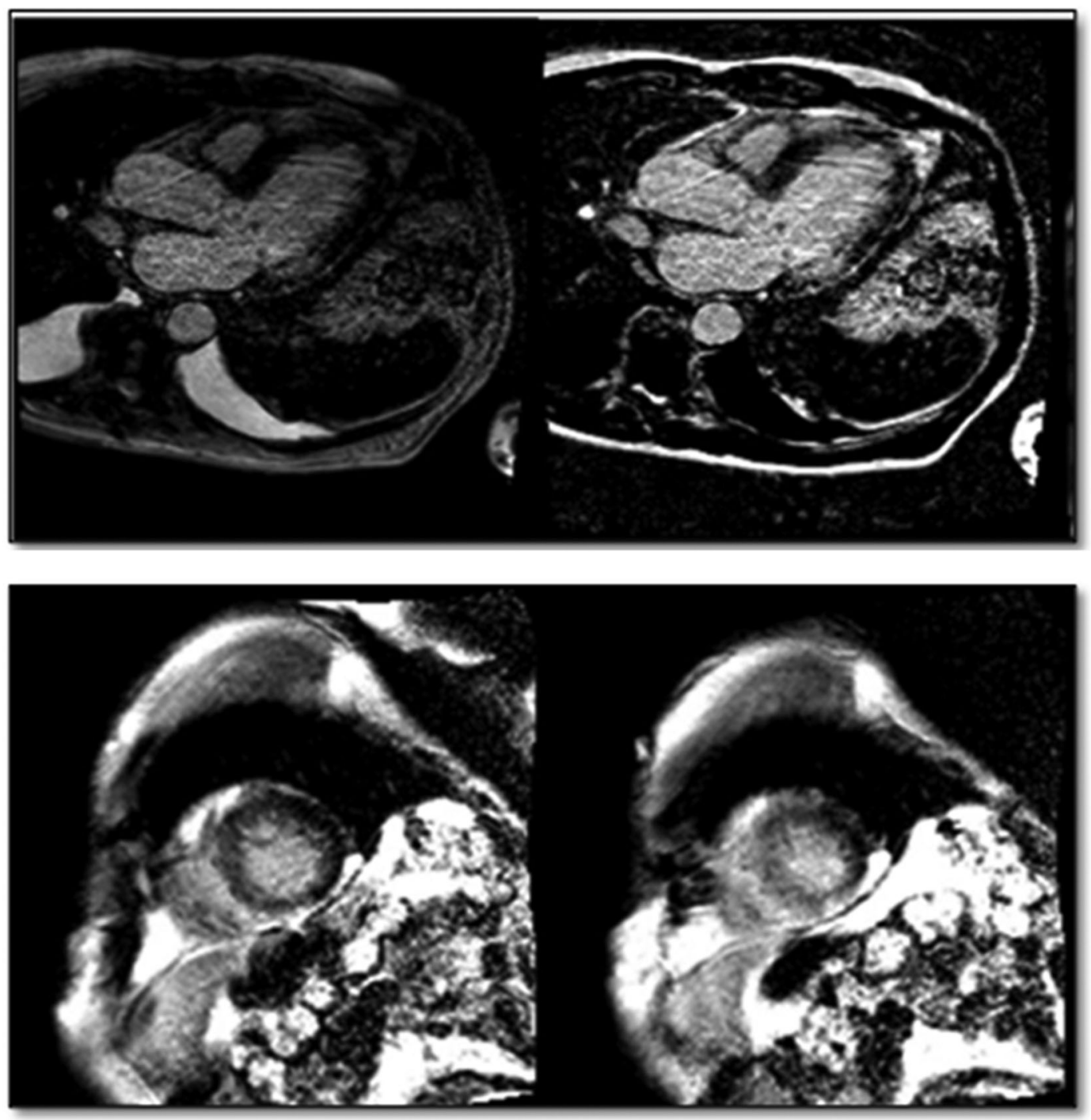

\section{Methods}

We searched for adverse drug reactions (ADRs) reported in VigiBase (NCTO3530215), the World Health Organization database containing individual case safety reports from more than 130 countries since $1967 .{ }^{5}$ VigiBase is managed by the Uppsala Monitoring Centre (Uppsala, Sweden). The use of confidential, electronically processed patient data was approved by the French National Commission for Data Protection and Liberties (Commission Nationale de I'Informatique et des Libertés; reference number 1922081). Using the MedDRA term 'cardiac disorders' (System Organ
Class level), we searched for CV adverse events associated with sipuleucel-T versus all other drugs in the database. Disproportionality analysis was calculated by the information component (IC), a Bayesian disproportionality indicator, which has been previously described. ${ }^{6}$ A positive $I_{025}$ (IC $95 \%$ lower end credibility interval) value $(>0)$ is the traditional threshold used in statistical signal detection at the Uppsala Monitoring Centre. To identify the co-occurrence of relevant CV-ADR associated with sipuleucel-T $\left(\mathrm{IC}_{025}>0\right)$, individual extracted cases were reviewed and the UpSet technique ${ }^{7}$ was used to visualize set intersections. 
Figure 3 Endomyocardial biopsy of the right ventricular septum. (A) Haematoxylin and eosin staining of myocardium (low magnification) showing several foci of lymphocyte infiltration. Scale bar, $100 \mu \mathrm{m}$. (B) Haematoxylin and eosin staining (high magnification) showing small mature lymphocytes infiltrating in the interstitial area. Scale bar, $50 \mu \mathrm{m}$. (C) Congo red staining showing no amyloid deposition. Inset: positive control. Scale bar, $50 \mu \mathrm{m}$. (D-F) Immunohistochemistry for CD4 (D), CD8 (E), and programmed cell death ligand-1 (F). Infiltrating lymphocytes are positive for CD4 or CD8, and negative for programmed cell death ligand-1. Scale bar, $50 \mu \mathrm{m}$.
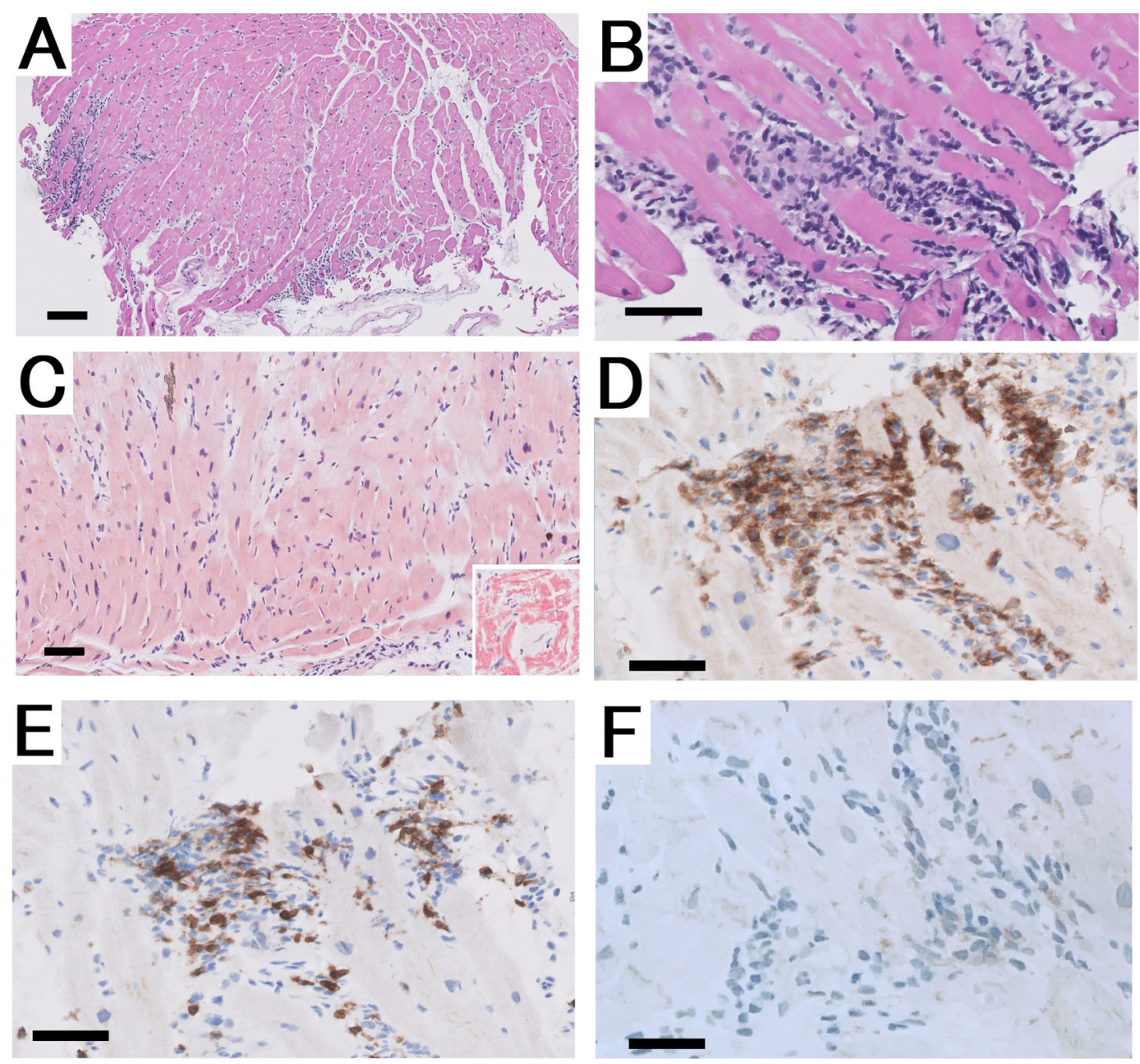

\section{Results}

The total number of CV-ADR reported with sipuleucel-T versus all other drugs was 306 and 22980 104, respectively, on $10 / 25 / 2020$. There were 18 MedDRA terms of 'cardiac disorders' associated with sipuleucel-T (positive $\mathrm{IC}_{025}>0$ ) (Table 1). MedDRA preferred terms levels were grouped into major CV-ADR categories where there were significant reports of myocardial ischemia, supraventricular tachycardias (SVTs) (atrial fibrillation/atrial flutter), congestive HF (CHF), and valvular disorders. All of these CV-ADRs were considered severe. Myocardial ischemia included acute myocardial infarction $\left(\mathrm{IC}_{025}=2.3\right)$ with $n=4 / 26(15 \%)$ of these individual case safety reports considered fatal. Among patients with 'cardiac failure congestive' $\left(\mathrm{IC}_{025}=1.4\right), 11$ of these 43 cases (26\%) were fatal with $42(98 \%)$ of these cases suspected to be solely due to sipuleucel-T. Other CV-ADRs were not significantly associated with sipuleucel-T (Table 1). Among the major CV-ADR categories, these occurred independently with minimal overlap among each major CV-ADR category. Myocardial ischemia occurred independently in $84.4 \%$ ( $n=65 / 77)$ of all cases, SVT $77.2 \%(n=44 / 57)$, CHF $75 \%$ ( $n=36 / 48)$, and valvular disorders $62.5 \%(n=5 / 8)$. SVT was rarely associated with $\mathrm{CHF}(n=6 / 48,12.5 \%)$ and myocardial infarction ( $n=6 / 77,7.79 \%$ ) (Figure 4).

\section{Discussion}

We describe the first reported phenomenon of a Tcell-mediated sipuleucel-T-induced cardiomyopathy. This is further corroborated by our pharmacovigilance analysis that 


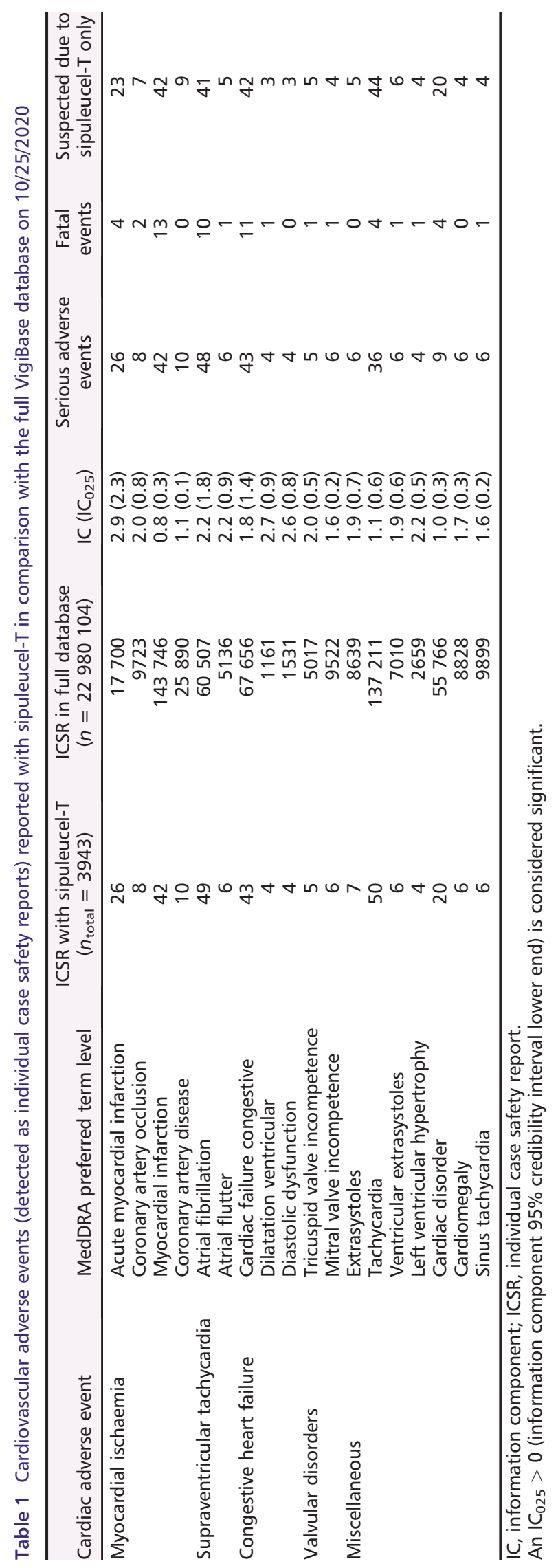


Figure 4 Intersection of major cardiovascular adverse drug reaction (CV-ADR) categories associated with sipuleucel-T. Myocardial ischemia occurred independently $84.4 \%(n=65 / 77)$ of all cases, supraventricular tachycardia (SVT) $77.2 \%(n=44 / 57)$, congestive heart failure (CHF) $75 \%(n=36 / 48)$, and valvular disorders $62.5 \%(n=5 / 8)$. SVT was rarely associated with CHF $(n=6 / 48,12.5 \%)$ and myocardial infarction $(n=6 / 77,7.79 \%)$.

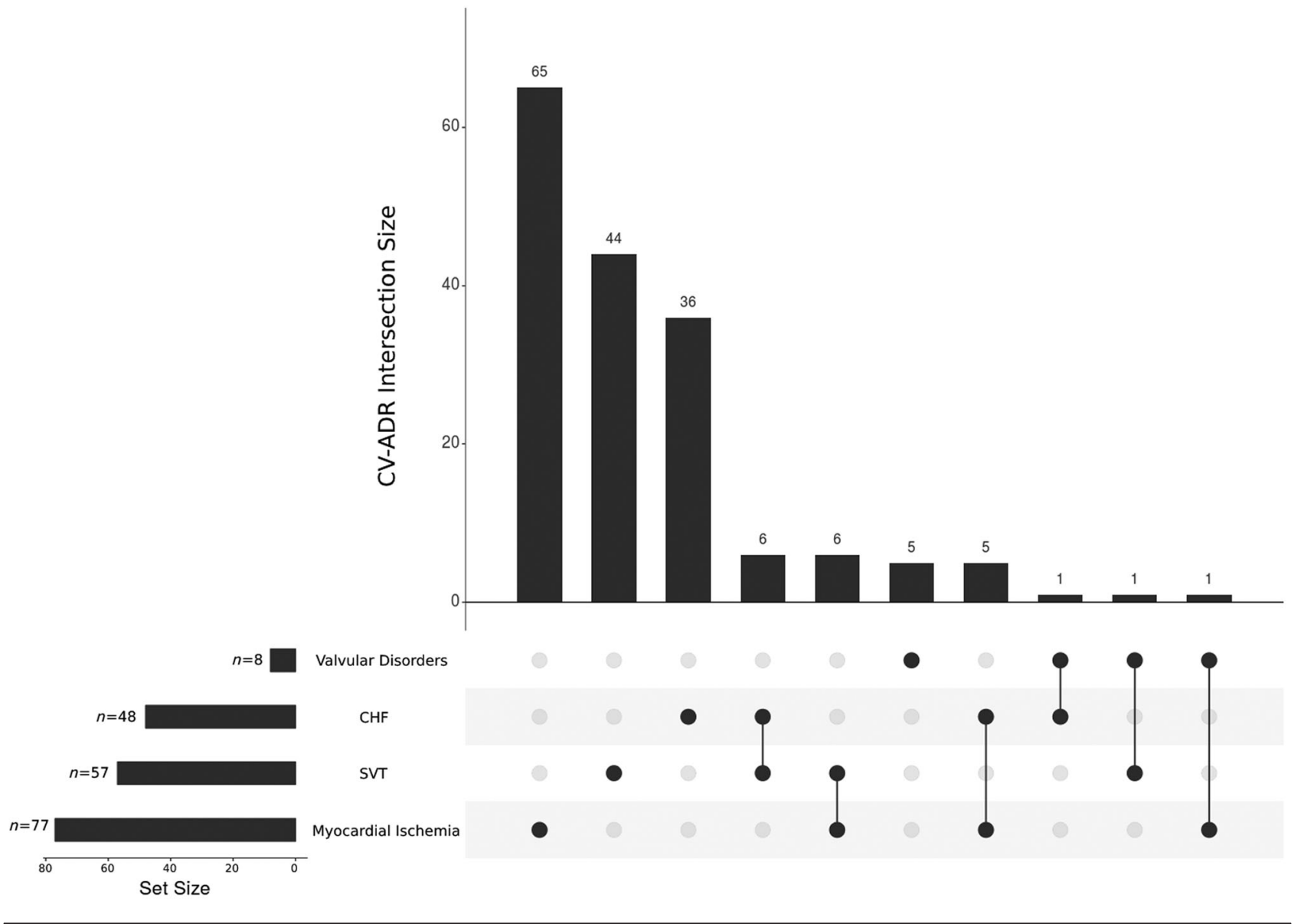

revealed an over-reporting of CHF with sipuleucel-T with $\sim 15 \%$ fatality.

Although CV toxicities from sipuleucel-T were uncommon among clinical trials, ${ }^{3}$ we demonstrate through VigiBase that there was significant over-reporting of myocardial ischemia, SVT, and CHF with sipuleucel-T versus all other drugs. While reports from VigiBase are non-homogeneous with the inability to definitively verify the cases with clinical, laboratory, or radiological tests, we suspect that these CV-ADRs may have been one of the manifestations of an inflammatory-mediated cardiomyopathy. ${ }^{8}$ Our observation of lymphocytic inflammation on myocardial biopsy suggests that sipuleucel-T invoked an inflammatory cascade that resulted in a T-cell-mediated cardiomyopathy with similar features to observed cases of immune-related $\mathrm{CV}$ toxicities such as with influenza vaccination $^{9,10}$ and $\mathrm{ICls}^{4}$

In fact, preclinical production studies of sipuleucel-T demonstrated an immune priming response followed by immune boosting with subsequent infusions, similar to classic vaccine-mediated cellular and humoral responses. ${ }^{11}$
Antigen-presenting cells, detected by CD54, were significantly up-regulated after the first dose with the most robust response at week 4, along with antigen-presenting cell activation-associated cytokines, which is followed by T-cell activation-associated cytokines in the second and third infusion preparations. ${ }^{11}$ Particularly, from peripheral blood samples obtained from patients receiving sipuleucel-T, both T-cell helper 1 (interferon and tumour necrosis factor- $\alpha$ ) and 2 [interleukin (IL)-5 and IL-13]-specific cytokines were elevated, supporting the mechanism of sipuleucel-T-mediated cellular and humoral responses. Acting in concert with interferon and tumour necrosis factor- $\alpha$, an increase in IL-17 levels was also observed, which has been implicated in the pathophysiology of autoimmune diseases. ${ }^{12}$ Specific responses measured by anti-PA2024 and anti-PAP antibody titres were sustained at least until week $26 .^{11}$ This may have resulted in cytokine cascade, ${ }^{9,10}$ which resulted in T-cell recruitment and myocardial injury in our patient.

Unique to our case was the observation of $\mathrm{CD} 4^{+}$and $\mathrm{CD} 8^{+}$ $T$ cells within focal inflammatory areas on myocardial biopsy. 
Notably, sipuleucel-T increases the frequency of $\mathrm{CD}^{+}$and $\mathrm{CD}^{+} \mathrm{T}$ cells within prostate tissue 2 to 3 weeks after treatment, particularly at the tumour margin. ${ }^{13}$ Whether sipuleucel-T may induce a generalized inflammatory response that can affect additional organs has not yet been reported in preclinical sipuleucel-T-treated animal models. ${ }^{13}$ These preclinical findings of an increased immune response at week 2 with the most robust response at week 4 are consistent with the timeline to development of our patient's inflammatory cardiomyopathy, further corroborating the high likelihood of sipuleucel-T exposure as the suspected cause.

We did not observe an increased expression of PD-L1 in the myocardium as previously demonstrated in $\mathrm{ICl}$-related myocarditis. ${ }^{4}$ This is likely due to a variation in the mechanism of action whereby $\mathrm{ICl}$ exhibits a generalized immune blockade whereas sipuleucel-T induces a destruction specific to prostate cancer cells. However, the phenomenon of 'antigen spread' with sipuleucel-T where additional prostate cancer-associated tumour antigens involved in PSA processing and oncologic downstream signalling were targeted has also been observed. ${ }^{14}$ We suspect that cross-reactivity and/or shared antigen recognition within the myocardium by sipuleucel-T is likely low, however possible in proinflammatory environments such as patients with $\mathrm{CV}$ risk factors. ${ }^{15}$

Our patient had LVEF recovery within 6 months on guideline-directed medical therapy only. This suggests that the immune-mediated mechanisms were likely transient, with reversion to a balanced non-inflammatory environment following completion of sipuleucel-T treatment. Nonetheless, our patient experienced a severe reaction reflecting our pharmacovigilance results in which approximately $15 \%$ of patients with spiuleucel-T-associated CHF associated suffered fatal outcomes. While our patient was concomitantly receiving androgen-deprivation therapy (ADT) with enzalutamide and leuprolide, the likelihood of ADT resulting in cardiomyopathy was low in the differential. Cardiotoxicities reported with ADT include cardiometabolic disturbances (hypertension, hyperglycemia, and hyperlipidemia) and risk of arrhythmias from prolonged $\mathrm{QTC}^{16}$ and are generally not associated risk of $\mathrm{HF}$; in clinical trials, $\mathrm{HF}$ was reported to be $<1 \% .{ }^{17,18}$ Docetaxel has also not been associated with HF despite prolonged use. ${ }^{19}$ Our patient tolerated ADT and docetaxel for several months but developed acute onset of symptoms with sipuleucel-T, correlating with the timeline of the immune modulating mechanism of sipuleucel-T and potential crosstalk between pathways making sipuleucel-T the most plausible culprit of our patient's inflammatory cardiomyopathy.

In comparison with the familiarity of cardiotoxicities associated with anthracyclines and anti-human epidermal growth factor receptor (HER2) therapy, prostate cancer therapy-induced cardiac adverse events are underrecognized. There have been concerns regarding adequate reporting and potential under recognition of adverse cardiac events with prostate cancer therapies as the studies were not designed to detect cardiotoxicities. ${ }^{20}$ Therefore, we encourage consideration of monitoring in patients with $\mathrm{CV}$ risk factors with serial electrocardiograms during weekly infusions and obtaining a baseline echocardiogram in patients receiving sipuleucel-T. Additional studies investigating the complex immune-mediated mechanisms in patients receiving immunotherapy and optimal management to avoid off-target organ inflammation without affecting the salutary effects of immunotherapy are highly warranted.

\section{Limitations}

Our study is not without limitations. As there are no current guidelines regarding obtaining baseline cardiac diagnostic testing prior to initiation of prostate cancer treatment, our patient did not have a baseline echocardiogram for comparison. The patient did not have any cardiac symptoms or complaints that would have suggested baseline systolic or valvular dysfunction; therefore, it is assumed that his baseline cardiac function was normal prior to and during his non-immunotherapy treatment for mCRPC. Another limitation of our study was the lack of viral genome data from EMB. The patient did not have any viral or infectious symptoms at the time of his admission in addition to negative blood cultures and respiratory viral panel; an infectious or viral-associated cardiomyopathy was very low in the differential. In addition, limitations with VigiBase reporting are voluntary, and thus, not all data fields are included in every report, and quality might be variable.

\section{Acknowledgements}

The supplied data from VigiBase ${ }^{\circledR}$ come from various sources. The likelihood of a causal relationship is not the same in all reports. The information does not represent the opinion of the World Health Organization.

\section{Conflict of interest}

None declared.

\section{Supporting information}

Additional supporting information may be found online in the Supporting Information section at the end of the article.

Figure S1. Supporting Information.

Figure S2. Supporting Information. 


\section{References}

1. Cheever M, Higano C. PROVENGE (sipuleucel-T) in prostate cancer: the first FDA-approved therapeutic cancer vaccine. Clin Cancer Res 2011; 17: 3520-3526.

2. Kantoff PW, Higano CS, Shore ND, Berger ER, Small EJ, Penson DF, Redfern CH, Ferrari AC, Dreicer R, Sims RB, Xu Y, Frohlich MW, Schellhammer PF. Sipuleucel-T immunotherapy for castration-resistant prostate cancer. $N$ Engl J Med 2010; 363: 411-422.

3. Higano CS, Armstrong AJ, Sartor AO, Vogelzang NJ, Kantoff PW, McLeod DG, Pieczonka CM, Penson DF, Shore ND, Vacirca J, Concepcion RS, Tutrone RF, Nordquist LT, Quinn DI, Kassabian V, Scholz MC, Harmon M, Tyler RC, Chang NN, Tang H, Cooperberg MR. Real-world outcomes of sipuleucel-T treatment in PROCEED, a prospective registry of men with metastatic castration-resistant prostate cancer. Cancer 2019; 125: 4172-4180.

4. Johnson DB, Balko JM, Compton ML, Chalkias S, Gorham J, Xu Y, Hicks M, Puzanov I, Alexander MR, Bloomer TL, Becker JR, Slosky DA, Phillips EJ, Pilkinton MA, Craig-Owens L, Kola N, Plautz G, Reshef DS, Deutsch JS, Deering RP, Olenchock BA, Lichtman AH, Roden DM, Seidman CE, Koralnik IJ, Seidman JG, Hoffman RD, Taube JM, Diaz LA Jr, Anders RA, Sosman JA, Moslehi JJ. Fulminant myocarditis with combination immune checkpoint blockade. $N$ Engl $J$ Med 2016; 375: 1749-1755.

5. Lindquist M. VigiBase, the WHO global ICSR database system: basic facts. Drug Inf $J$ 2008; 42: 409-419.

6. Salem JE, Manouchehri A, Moey M, Lebrun-Vignes B, Bastarache L, Pariente A, Gobert A, Spano JP, Balko JM, Bonaca MP, Roden DM, Johnson DB, Moslehi JJ. Cardiovascular toxicities associated with immune checkpoint inhibitors: an observational, retrospective, pharmacovigilance study. Lancet Oncol 2018; 19: 1579-1589.
7. Lex A, Gehlenborg N, Strobelt $\mathrm{H}$, Vuillemot $R$, Pfister $H$. UpSet: visualization of intersecting sets. IEEE Trans Vis Comput Graph 2014; 20: 1983-1992.

8. Lyon AR, Yousaf N, Battisti NML, Moslehi J, Larkin J. Immune checkpoint inhibitors and cardiovascular toxicity. Lancet Oncol 2018; 19: e447-e458.

9. Saraiya N, Singh S, Corpuz M. Fatal influenza myocarditis with incessant ventricular tachycardia. BMJ Case Rep CP 2019; 12: e228201.

10. Kim YJ, Bae JI, Ryoo SM, Kim WY. Acute fulminant myocarditis following influenza vaccination requiring extracorporeal membrane oxygenation. Acute Crit Care 2019; 34: 165-169.

11. Sheikh NA, Petrylak D, Kantoff PW, dela Rosa C, Stewart FP, Kuan LY, Whitmore JB, Trager JB, Poehlein $\mathrm{CH}$, Frohlich MW, Urdal DL. Sipuleucel-T immune parameters correlate with survival: an analysis of the randomized phase 3 clinical trials in men with castration-resistant prostate cancer. Cancer Immunol Immunother 2013; 62: 137-147.

12. Kuwabara $T$, Ishikawa $F$, Kondo $M$, Kakiuchi T. The role of IL-17 and related cytokines in inflammatory autoimmune diseases. Mediators Inflamm 2017; 2017: 3908061.

13. Laus R, Yang DM, Ruegg CL, Shapero $\mathrm{MH}$, Slagle $\mathrm{PH}$, Small EJ, Burch $\mathrm{P}$, Valone FH. Dendritic cell immunotherapy of prostate cancer: preclinical models and early clinical experience. Cancer Res Ther Control 2001; 11: 1-10.

14. GuhaThakurta D, Sheikh NA, Fan LQ, Kandadi H, Meagher TC, Hall SJ, Kantoff PW, Higano CS, Small EJ, Gardner TA, Bailey K, Vu T, DeVries T, Whitmore JB, Frohlich MW, Trager JB, Drake CG. Humoral immune response against nontargeted tumor antigens after treatment with sipuleucel-T and its association with improved clinical outcome. Clin Cancer Res 2015; 21 . 3619-3630.
15. Willerson JT, Ridker PM. Inflammation as a cardiovascular risk factor. Circulation 2004; 109: II2-II10.

16. Salem JE, Yang T, Moslehi JJ, Waintraub X, Gandjbakhch E, Bachelot A, Hidden-Lucet F, Hulot JS, Knollmann BC, Lebrun-Vignes B, Funck-Brentano C, Glazer AM, Roden DM. Androgenic effects on ventricular repolarization: a translational study from the international pharmacovigilance database to iPSC-cardiomyocytes. Circulation 2019; 140: 1070-1080.

17. Scher HI, Fizazi K, Saad F, Taplin ME, Sternberg CN, Miller $\mathrm{K}$, de Wit $\mathrm{R}$, Mulders P, Chi KN, Shore ND, Armstrong AJ, Flaig TW, Fléchon A, Mainwaring P, Fleming M, Hainsworth JD, Hirmand M, Selby B, Seely L, de Bono JS. Increased survival with enzalutamide in prostate cancer after chemotherapy. $N$ Engl J Med 2012; 367: 1187-1197.

18. Beer TM, Armstrong AJ, Rathkopf DE, Loriot Y, Sternberg CN, Higano CS, Iversen P, Bhattacharya S, Carles J, Chowdhury S, Davis ID. Enzalutamide in metastatic prostate cancer before chemotherapy. $N$ Engl $J$ Med 2014; 371: 424-433.

19. Zamorano JL, Lancellotti P, Rodriguez Muñoz D, Aboyans V, Asteggiano R, Galderisi M, Habib G, Lenihan DJ, Lip GYH, Lyon AR, Fernandez TL, Mohty D, Piepoli MF, Tamargo J, Torbicki A, Suter TM, ESC Scientific Document Group. 2016 ESC Position Paper on cancer treatments and cardiovascular toxicity developed under the auspices of the ESC Committee for Practice Guidelines: the Task Force for cancer treatments and cardiovascular toxicity of the European Society of Cardiology (ESC). Eur Heart $J$ 2016; 37: 2768-2801.

20. Witteles RM, Telli M. Underestimating cardiac toxicity in cancer trials: lessons learned? J Clin Oncol 2012; 30: 1916-1918. 